\title{
The human larynx- an anatomical aspect: a review
}

\author{
Manish Munjal ${ }^{1 *}$, Hitant Vohra², Shubham Munjal², Ajay Kumar², Anu Sharma², \\ Sonia Singh ${ }^{2}$, Anshu Soni ${ }^{2}$, Seema Gupta ${ }^{2}$, Meenakshi Goel ${ }^{2}$
}

${ }^{1}$ Department of ENTHNS, ${ }^{2}$ Department of Anatomy, Dayanand Medical College, Ludhiana, Punjab, India

Received: 12 November 2020

Accepted: 15 December 2020

\section{*Correspondence:}

Dr. Manish Munjal,

E-mail: manishmunjaldr@yahoo.com

Copyright: (C) the author(s), publisher and licensee Medip Academy. This is an open-access article distributed under the terms of the Creative Commons Attribution Non-Commercial License, which permits unrestricted non-commercial use, distribution, and reproduction in any medium, provided the original work is properly cited.

\section{ABSTRACT}

The organ of phonation and the air conduit, the larynx of the humans with its intricate architecture has been elaborated in context to its unique musculature, vasculature, innervation and histological anatomy.

Keywords: Larynx, Cartilages, Musculature, Vascularity, Innervation

\section{INTRODUCTION}

Anatomist Hippocrates and Aristotle in 400 B.C. could not elucidate the anatomy and physiology of the "voice box". 500 years later, Galenos accurately described the delicate cartilages, muscles and nerves of the animal larynx. In the 16th century Andreas Vesalius further elaborated the macroscopic anatomy of the larynx. Jacob Henle 1838 based on his microscopic studies, described the different epithelia in the larynx. The literature has been reviewed in relation to various aspects- embryological, gross and the histological anatomy.

\section{REVIEW OF LITERATURE}

\section{Embryology of the larynx}

During the fourth week of embryonic development, the tracheobronchial diverticulum appears in the ventral wall of the primitive pharynx just below the hypobranchial eminence. The edges of this groove form the esophagealtracheal septum which fuses caudally, leaving a slit like aperture cranially into the pharynx. The cranial end of the tube forms the larynx and the trachea while the caudal end, the bronchi and the lungs. ${ }^{1}$

\section{Gross anatomy of the larynx}

The larynx extends from the laryngeal inlet to the inferior border of the cricoid cartilage. It extends from the third to sixth cervical vertebrae, being a little higher in women than in men. ${ }^{1}$ In males the average length is $44 \mathrm{~mm}$, transverse diameter $43 \mathrm{~mm}$ and antero-posterior diameter is $36 \mathrm{~mm}$. In females it is $36 \mathrm{~mm}, 41 \mathrm{~mm}$ and $26 \mathrm{~mm}$ respectively. The larynx is subdivided anatomically into the supraglottis, glottis and subglottis by the false and the true cords. The supra-glottis consists of superiorly the epiglottis and ary-epiglottic folds as they sweep down to the arytenoids. Its lower border is formed by the ventricular bands or false cords, which form the upper border of the glottis. The glottis includes the vocal cords and the anterior and posterior commissures. The demarcation between the glottis and sub glottis has been debated in literature and is either defined as at the level of vocal cords or $5-10 \mathrm{~mm}$ below. The sub glottis becomes the trachea at the lower border of cricoid. ${ }^{1}$ The skeletal framework of larynx is constituted by the thyroid bone and a number of cartilages connected by ligaments, membranes and the intrinsic and extrinsic muscles; which also provide it, the required stability. It is lined by a mucus membrane continuous above with the pharynx and below with that of the trachea. ${ }^{1}$ The epiglottis is a thin, leaf like sheet of elastic fibrocartilage which projects upwards behind the tongue and the body of the hyoid bone. It is attached inferiorly to the thyroid cartilage by the thyroepiglottic ligament and anteriorly to the hyoid bone 
by hyoepiglottic ligament. From either side of the epiglottis, the aryepiglottic folds pass down to the apex of the arytenoids. The mucous membrane overlying the epiglottis is reflected on to the base of the tongue, forming the glossoepiglottic fold in the midline and laterally the lateral glosso epiglottic folds. ${ }^{1}$ The epiglottis is not functionally developed in humans.

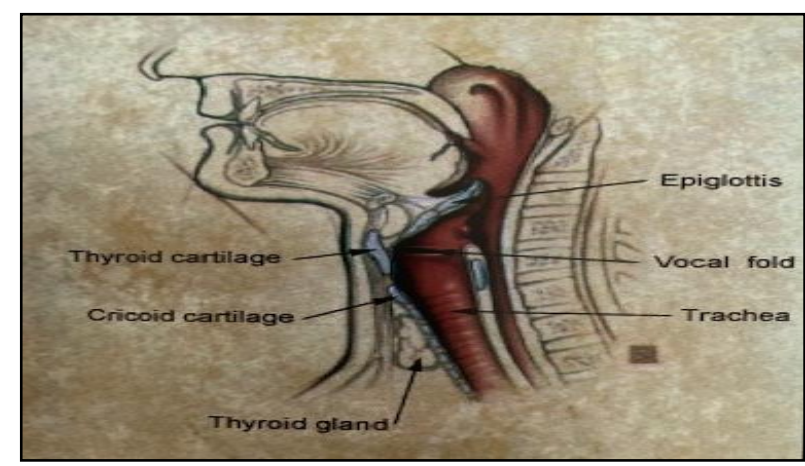

Figure 1: Sagittal section through the neck. ${ }^{1}$

In fact, respiration, deglutition and phonation can take place almost normally even if it has been removed. In neonates and infants, however, the epiglottis is omegashaped. This long, deeply grooved, floppy epiglottis closely resembles that of aquatic mammals and is more suited to its function of protecting the nasotracheal air passage during suckling. ${ }^{2}$ The thyroid cartilage is a shield like cartilage and is the longest of the laryngeal cartilages. It is composed of two lamina fused in the midline anteriorly giving rise to a laryngeal prominence. The angle of fusion is $90^{\circ}$ in men and $120^{\circ}$ in women. ${ }^{2}$ The posterior border of each lamina is elongated to form the superior and inferior cornua. On the external surface of each lamina, an oblique line curves downwards and forwards from the superior thyroid tubercle, situated just in front of root of the superior horn, to the inferior thyroid tubercle on the lower border of the lamina. This line marks the attachment of the thyrohyoid, sternothyroid and inferior constrictor muscles. On the inner aspect of the thyroid cartilage just below the thyroid notch in the midline is attached the thyroepiglottic ligament and below this and on each side of the midline, the vestibular and vocal ligaments, as well as thyroarytenoid, thyroepiglottic and vocalis muscles. The fusion of the anterior end of the two vocal ligaments produces the anterior commissure tendon. ${ }^{1}$ The cricoid cartilage is the only complete cartilaginous ring encircling the airway, forming the inferior part of the anterior and lateral walls and most of the posterior wall of the larynx. It has a deep broad lamina posteriorly and a narrow arch anteriorly with a facet for articulation with the inferior cornua of the thyroid cartilage Weir, 1997. ${ }^{2}$ The upper and lateral borders of the cricoid lamina have articular facets for the arytenoids, Strome et al, $1985 .^{3}$

The arytenoid cartilages are irregular, three sided pyramids with an upward projection. To the anterior projection the vocal process, attach the vocal folds and to the lateral projection, the muscular process, attach the posterior cricoarytenoid and lateral cricothyroid muscles. Between these two, the anterolateral surface is divided into fossa by a crest. The triangular fossa gives attachment to the vestibular ligament and to vocalis and lateral cricoarytenoid muscles. The medial surfaces are covered with mucus membrane and form the lateral boundary of the posterior glottis. The corniculate and cuneiform elastic fibrocartilages are embedded in the posterior part of the aryepiglottic folds of the mucous membrane. ${ }^{1}$

The extrinsic ligaments of the larynx connect the laryngeal cartilages to the hyoid above and trachea below. These are grouped as median thyrohyoid ligament, lateral thyrohyoid ligament, cricotracheal ligament and hyoepiglottic ligament. The thyrohyoid ligament is pierced by the internal branch of superior laryngeal nerve and by the superior laryngeal vessels. ${ }^{1}$

The intrinsic ligaments connect the laryngeal cartilages together; strengthen the capsule of the intercartilaginous joints. They form a broad sheet of fibroelastic tissue, the fibroelastic membrane, which lies beneath the mucous membrane of larynx forming an internal framework. The fibroelastic membrane is divided into an upper and lower part by the laryngeal ventricle. ${ }^{1}$

Upper quadrilateral membrane. Its upper end forms the aryepiglotic fold and lower margin thickens to form vestibular ligament.

Lower crico vocal membrane or conus elasticus. Its free upper border forms the vocal ligament.

\section{Glottis}

The glottis lies between the false and true vocal cords which cover the vestibular and vocal ligaments respectively. The vestibular folds are two thick folds of mucous membrane each enclosing a narrow band of fibrous tissue, the vestibular ligament, which is the lower border of the upper quadrilateral membrane. It is fixed in front at the angle of the thyroid cartilage just below the attachment of the epiglottic cartilages and behind to the antero-lateral surface of the arytenoid cartilage just above the vocal process. The vocal folds extend from the middle of the angle of the thyroid cartilage to the vocal process of the arytenoid cartilages and underneath is the upper border of the conus elasticus. ${ }^{1}$

The anterior three-fifths of the vocal cord are between the vocal folds and are called the inter-membranous part of the cord. The remaining two-fifths posteriorly are between the vocal processes of the arytenoid and are called intercartilaginous portion. The fissure between the vestibular folds (false vocal cords) is the rima vestibuli and 'between the true vocal cords is rima glottis. At rest it is $8 \mathrm{~mm}$ apart. In males it is $23 \mathrm{~mm}$ in length and in females $16 \mathrm{~mm}$. $^{1}$

\section{The laryngeal musculature}


The shape of vocal folds and movement are primarily the result of intrinsic laryngeal muscle activity. Extrinsic laryngeal muscles too affect vocal fold shape and movement but to a lesser degree. The muscles that adduct or close the vocal folds are the lateral cricoarytenoid, thyroarytenoid, and interarytenoid muscles. The posterior cricoarytenoid muscle is the only muscle that abducts or opens the vocal folds. The cricothyroid muscle lies in the gap between the thyroid and cricoid cartilages, thereby stretching cords. Professional singers rely particularly on proper control of the cricothyroid muscle to attain a higher pitch during their performance. ${ }^{4}$

\section{Extrinsic muscles}

These anchor the larynx to adjacent structures and maintain its position in the neck. ${ }^{1,4}$ These are divided into suprahyoid and infrahyoid group of muscles.

Suprahyoid muscles include mylohyoid, geniohyoid, stylohyoid, stylopharyngeus, palatopharyngeus and salpingopharyngeus.

Infrahyoid muscles are thyrohyoid, sternothyroid, sternohyoid and omohyoid.

\section{Intrinsic muscles}

These muscles move the various cartilages of the larynx. They alter the mechanical properties of the vocal folds, their position, shape, elasticity and viscosity of each layer of the vocal fold. ${ }^{1,4}$ These are divided into:

Openers of glottis - (Abductors)- Posterior cricoarytenoid

Closures of glottis (Adductors)- Lateral cricoarytenoid Transverse arytenoid - Oblique arytenoid

Tensors of vocal ligament- Thyroarytenoid - Cricothyroid - Vocalis

After the shape of laryngeal inlet - Aryepiglotticus Thyroepiglotticus.

\section{Spaces within the larynx}

\section{Pre-epiglottic space}

It is a wedge-shaped space with the point of wedge directed inferiorly. It is also called as the Space of Boyer. ${ }^{4}$ Anteriorly it is bounded by the thyrohyoid ligament and the hyoid bone and posteriorly by the epiglottis. Superiorly, the hyoepiglottic ligament connects the epiglottis to the hyoid bone. The pre epiglottic space communicates laterally with the paraglottic space on either side. $^{1}$

\section{Para glottic space}

It is bounded by the thyroid cartilage laterally, conus elasticus and quadrangular membrane medially and posteriorly by the piriform fossa. It encompasses the laryngeal ventricles and saccules. ${ }^{1}$

\section{Reinke's Space}

Reinke, 1895 described the existence of loose sub epithelial connective of the vocal cords, the presence of which permits wave like motion of mucosa during phonation. This was referred to as laryngeal bursa by Pressman, 19565. This potential space is limited above and below by the superior and inferior arcuate lines, by the junction of squamous with respiratory epithelium. Anteriorly, anterior commissure and posteriorly the tip of the vocal process of the arytenoid bounds it. The squamous epithelium of the vocal cords and the elastic tissue of the vocal ligament represent the superficial and deep boundaries of the space. It has an inadequate lymphatic blood supply hence products may accumulate in it without resolution, giving rise to tissue reaction.

\section{Vascularity of larynx}

\section{Arterial supply}

It is derived from the laryngeal branches of the superior thyroid artery i.e. branch of external carotid artery and the inferior thyroid artery i.e. branch of the thyrocervical trunk of the first part of subclavian artery and the cricothyroid artery i.e. branch of superior thyroid artery.

\section{Venous drainage}

It accompanies the arteries. The superior laryngeal, veins draining into internal jugular vein via superior thyroid or facial vein and inferior laryngeal veins draining into brachiocephalic vein via inferior thyroid vein. ${ }^{1}$

\section{Nerve supply of larynx}

The innervation is by the superior and recurrent laryngeal branches of vagus nerve.

\section{The superior laryngeal nerves}

The external branch of the superior laryngeal nerve provides motor supply to the cricothyroid muscle while the internal branch divides into two main sensory and secretomotor branches. The superior laryngeal nerve ends by anastomosing with an ascending branch of the recurrent laryngeal nerve i.e. Galen's anastomosis.

\section{The recurrent laryngeal nerve}

The recurrent laryngeal nerve too divides into motor and sensory branches to supply all the intrinsic muscles of larynx, except the cricothyroid and laryngeal mucosa below the level of vocal folds. Damage to recurrent 
laryngeal nerve causes hoarseness clinically and medialization of the arytenoid cartilage radiographically. ${ }^{1}$

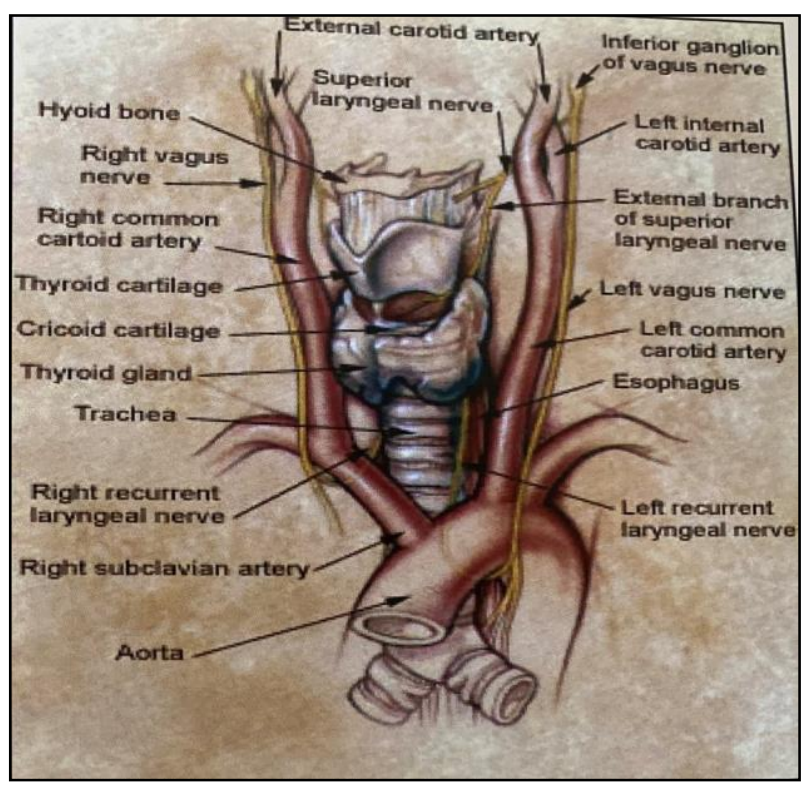

Figure 2: Innervation of the larynx. ${ }^{1}$

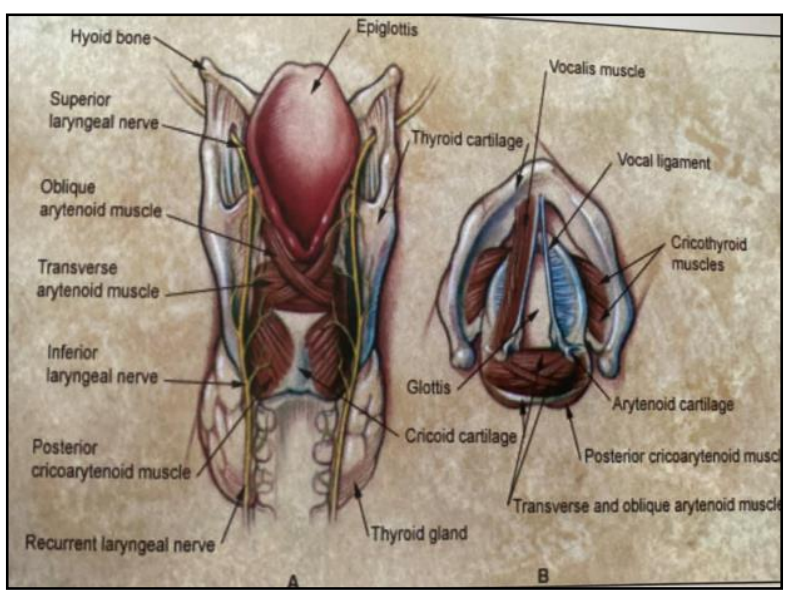

Figure 3: Cross section larynx from posterior and above. ${ }^{1}$

\section{Lymphatic drainage of larynx}

The lymphatics of larynx are separated by the vocal folds into an upper and 3 lower group. There are two sets of lymphatics.

Superficial or intramucosal, interlacing between the two sides of the larynx.

Deep or sub mucosal, independent of either half of the larynx, Pressman et al, 1956. ${ }^{5}$ The vocal folds are firmly adherent to the underlying vocal ligaments, hence have no lymphatics, which account for the clearly defined watershed between the upper and lower zones. The upper zone drains into the upper deep cervical nodes via the lymph vessels which accompany the superior laryngeal blood vessels. The lower zone drains via the pretracheal and prelaryngeal nodes into the lower deep cervical group. The drainage is ipsilateral but in the subglottic space it is bilateral.

\section{Histological anatomy of vocal folds}

The medial belly of the thyroarytenoid known as the vocalis muscle forms the body of the vocal fold. The thin, lubricated epithelium covering the vocal folds forms the area of contact between the two vibrating vocal folds and acts like a capsule, maintaining the shape of the vocal folds. This epithelium lines most of the vocal tract and is pseudo-stratified, ciliated columnar epithelium; a typical respiratory epithelium involved in handling mucous secretions. The vibratory margin of the vocal fold is covered by stratified squamous epithelium, to withstand trauma of opposing vocal fold contact. The superficial layer of the lamina propria known as Reinke's space consists primarily of loose fibers and matrix. The intermediate layer of lamina propria consists primarily of elastic fibers devoid of fibroblasts. The deep layer of lamina propria is composed primarily of collagenous fibers rich in fibroblasts. The intermediate and deep layers of the lamina propria constitute the vocal ligament and lie immediately below the Reinke's space, Hirano M,1977. ${ }^{6}$ This arrangement permits change of stiffness gradually from pliable membranous vocal fold to a stiff vocal cord resembling the thyroid cartilage. Hirano M, 1993. ${ }^{7}$ BIood vessels in the vibratory margin come from posterior and anterior origins and run parallel to the vibratory margin. The vibratory margin contains no glands, which are likely to interfere with the smoothness of vibratory waves. ${ }^{8}$ The entire mucous membrane is richly supplied by mucous which facilitates lubrication. These glands are beneath the mucosal lining and are tubo-alveolar type which produce mixed seromucinous secretions. Nassar et al 1971. ${ }^{9}$ Microvilli and micro ridges are present on the surface cells of the vocal fold epithelium and elsewhere in the larynx, Andrews 1975, Tillman et al 1977. ${ }^{10,11}$

The five layers have different mechanical properties like ball bearings of different sizes that permit the smooth shearing action essential for proper vocal fold vibration. Mechanically, the vocal fold structures act more like three layers consisting of the cover (epithelium and Reinke's space), transition (intermediate and deep layers of the lamina propria), and the body (the vocalis muscles). 8The vocal folds may be considered as the oscillators of the vocal mechanism. ${ }^{12}$

The vocal fold histologic characteristics are fairly consistent along the length of the vocal fold with a few exceptions. At the anterior-most portion of the vocal fold there is a mass of collagenous fibers known as the anterior commisure tendon or Broyle's tendon. These fibers are attached to the inner thyroid perichondrium. The intermediate layer of the lamina propria is thickened at the anterior and posterior ends of the membranous vocal fold. These regions are known as the anterior and posterior 
macula flava. These structures serve as a transition zone between the stiff thyroid and arytenoid cartilage and the pliable membranous vocal fold. ${ }^{4}$ These structures may fashion the vocal folds and provide protection from mechanical damage by vibration. ${ }^{13}$ The vocal fold epidermis is secured to the superficial "lamina propria through the basement membrane zone, Gray SD, 2000. ${ }^{15}$ Any disruption in this anchoring system can have serious consequences in voice production. A person with fewer anchoring fibers might be predisposed to the development of vocal fold nodules. The lamina propria contains fibroblasts, myofibroblasts, and macrophages; the presence of which indicates that the human vocal fold is constantly going through a cycle of minor injury and repair. However, the myofibroblasts must be given time to perform this repair. If a patient develops a minor injury because of overuse, the voice must be rested to allow the myofibroblasts perform needed repairs. This typically happens in 2 to 3 days. ${ }^{4}$ The noncellular or extracellular component of the lamina propria contains fibrous and interstitial proteins like collagen and elastin. ${ }^{14,15}$ Collagen provides strength and structure to the Vocal fold. Elastin provides elasticity to vocal fold to deform and then return to its original shape. Interstitial proteins affect tissue viscosity to provide dampening or shock-absorbing ability. ${ }^{16}$ With aging, the extracellular matrix turnover slows and older collagen undergoes a process known as cross-linking. ${ }^{4}$ Cross-linking results in less elastic elastin and stiffer collagen. ${ }^{17,18}$ As a result, the vocal folds become less elastic and stiffer as the human body ages).

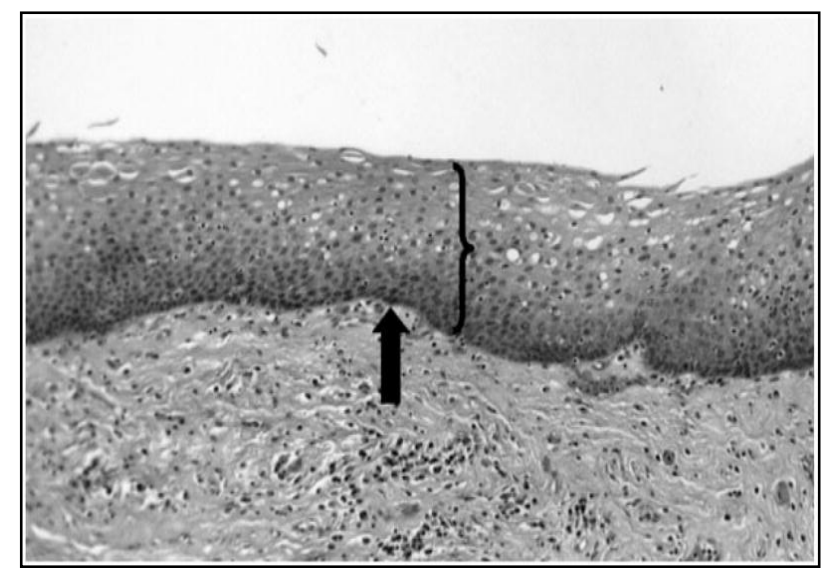

Figure 4: True vocal cord histological section under light microscopy. ${ }^{19}$

\section{CONCLUSION}

Individuals have a unique voice that is determined by the peculiar characteristics of their larynges. The fine neuromuscular coordination is vital to this voice production and affects the airflow to the lower respiratory tract.

Funding: No funding sources Conflict of interest: None declared Ethical approval: Not required

\section{REFERENCES}

1. Beasly N. Anatomy of larynx and tracheobronchial tree. Scott Brown's Otorhinolaryngology, Head and Neck Surgery 7th Ed 2008;2(162):2130-44.

2. Weir N. Anatomy of the larynx and tracheobronchial tree. Scott- Brown's Otolaryngology; ButterworthHeinemann Linacre House, Jordon Hill, Oxford, U.K. 6th Edition. 1997;1:1-28.

3. Strome M, Kelly JH, Fried MP. Manual of Otolaryngology. Little, Brown and Company. Boston 1st Ed. 1985;145.

4. Noordzij JP, Ossoff RH. Anatomy and physiology of the larynx. Otolaryngologic Clinics of North America. 2006;39(1):1-0.

5. Pressman J, Dowdy A, Libby R, Fields M. LXXXI Further Studies upon the Submucosal Compartments and Lymphatics of the Larynx by the Injection of Dyes and Radioisotopes. Annals of Otology, Rhinology \& Laryngology. 1956;65(4):963-80.

6. Hirano M. Structure and vibratory behavior of the vocal fold. Dynamic aspects of speech production. 1977;13-30.

7. Hirano M. Surgical anatomy and physiology of the vocal folds. Voice surgery. St.Louis (MO): MosbyYear Book. 1993;135-58.

8. Sataloff RT, Heman-Ackah YD, Hawkshaw MJ. Clinical anatomy and physiology of the voice. Otolaryngologic clinics of North America. 2007;40(5):909-29.

9. Nassar VH, Bridger GP. Topography of the laryngeal mucous glands. Archives of Otolaryngology. 1971;94(6):490-8.

10. Andrews PM. microplicae-morphology, distribution, origin and possible functional significance. Journal of cell biology. 1975;67(2)111.

11. Tillman B, Peitzch -Rohrscheider I, Hoenges HL. The human vocal cords surface .cell tissue research (berlin). 1977;185:279-283.

12. Swerdlin Y, Smith J, Wolfe J. The effect of whisper and creak vocal mechanisms on vocal tract resonances. The Journal of the Acoustical Society of America. 2010;127(4):2590-8.

13. Hirano M, Sato K, Nakashima T. Fibroblasts in human vocal fold mucosa. Acta oto-laryngologica. 1999;119(2):271-6.

14. Gray SD, Titze IR, Chan R, Hammond TH. Vocal fold proteoglycans and their influence on biomechanics. The Laryngoscope. 1999;109(6):84554.

15. Gray SD, Hammond E, Hanson DF. Benign pathologic responses of the larynx. Annals of Otology, Rhinology \& Laryngology. 1995;104(1):13-8.

16. Balazs EA. The rheological properties and biological function of hyaluronic acid. Chemistry and molecular biology of the intercellular matrix. 1970;3:1241-53.

17. Niewoehner DE, Kleinerman JE, Liotta LA. Elastic behavior of postmortem human lungs: effects of 
aging and mild emphysema. Journal of applied physiology. 1975;39(6):943-9.

18. Schnider SL, Kohn RR. Effects of age and diabetes mellitus on the solubility of collagen from human skin, tracheal cartilage and dura mater. Experimental Gerontology. 1982;17(3):185-94.

19. Kaiser ML, Rubinstein M, Vokes DE, Ridgway JM, Guo S, Gu M, Crumley RL, Armstrong WB, Chen Z, Wong BJ. Laryngeal epithelial thickness: a comparison between optical coherence tomography and histology. Clin Otolaryngol. 2009;34(5):460-6.

Cite this article as: Munjal M, Vohra H, Munjal S, Kumar A, Sharma A, Singh S et al. The human larynx -an anatomical aspect: a review. Int J Otorhinolaryngol Head Neck Surg 2021;7:207-12. 